\title{
Changes in cardiac repolarization during clinical episodes of nocturnal hypoglycaemia in adults with Type 1 diabetes
}

\author{
R. T. C. E. Robinson ${ }^{1}$ N. D. Harris ${ }^{2}$ R. H. Ireland ${ }^{1}$ I. A. Macdonald ${ }^{3}$ S. R. Heller ${ }^{1,4}$ \\ ${ }^{1}$ University of Sheffield, UK \\ 2 Royal Hallamshire Hospital, Sheffield, UK \\ ${ }^{3}$ University of Nottingham, Nottingham, UK \\ ${ }^{4}$ Northern General Hospital, Sheffield, UK
}

\section{Abstract}

Aims/hypothesis. Experimental hypoglycaemia leads to abnormal cardiac repolarization manifest by a lengthened QT interval and caused by adrenergic stimulation. However it is less clear whether spontaneous clinical episodes lead to similar changes. We have therefore measured cardiac ventricular repolarization and counterregulatory responses in patients with Type 1 diabetes during hypoglycaemic and euglycaemic nights.

Methods. We studied 22 patients with Type 1 diabetes (mean age $40.4 \pm 17.2$ years, duration of diabetes $17.2 \pm 9.3$ years, $\mathrm{HbA} 1 \mathrm{c} 8.2 \pm 1.2 \%$ overnight). Measurements were taken hourly of blood glucose, plasma potassium, catecholamines and high resolution electrocardiograms.

Results. Hypoglycaemia (blood glucose level $<2.5 \mathrm{mmol} / \mathrm{l}$ ) occurred on 7 of the 22 nights. During overnight hypoglycaemia, QTc interval increased by $27 \mathrm{~ms}( \pm 15)$ above baseline, compared with $9 \mathrm{~ms}$
$( \pm 19)$ during nights with no nocturnal hypoglycaemia $(p=0.034,95 \% \mathrm{CI} 2,35)$. Adrenaline increased by $0.33 \mathrm{nmol} / \mathrm{l}( \pm 0.21)$ above baseline during hypoglycaemia, compared with $-0.05 \mathrm{nmol} / \mathrm{l}( \pm 0.08)$ during euglycaemia $(p=0.001,95 \% \mathrm{CI} 0.19,0.56 \mathrm{nmol} / \mathrm{l})$. There was no significant difference between potassium, and noradrenaline concentrations between the two groups. Conclusion/interpretation. QTc interval lengthens significantly during spontaneous nocturnal hypoglycaemia. Increases are generally less than those observed during experimental hypoglycaemia and could reflect attenuated sympathoadrenal responses during clinical episodes. The clinical relevance of these changes is uncertain but is consistent with the hypothesis that clinical hypoglycaemia can cause abnormal cardiac repolarization and an attendant risk of cardiac arrhythmia. [Diabetologia (2004) 47:312-315]

Keywords Ventricular repolarization - QT interval, Type 1 diabetes mellitus · Overnight euglycaemia · Hypoglycaemia
The Long QT syndrome describes a variety of conditions characterised by a lengthened QT interval on the surface ECG reflecting abnormal cardiac repolarization. Its clinical importance is due to its association

Received: 13 August 2003 / Revised: 9 October 2003

Published online: 8 January 2004

(C) Springer-Verlag 2004

S. R. Heller (-)

Northern General Hospital, Sheffield, S5 7AU, UK

E-mail: S.Heller@sheffield.ac.uk

Abbreviations: ECG, electrocardiogram with torsades de pointes ventricular tachycardia and sudden death $[1,2]$. The critical QT period which confers particular vulnerability to VT probably varies for different individuals but is considered to be around $550 \mathrm{~ms}$ [3]. We have previously shown that experimental hypoglycaemia is an acquired cause of abnormal cardiac repolarization with some individuals experiencing corrected QT intervals up to $580 \mathrm{~ms}[4,5]$. Our data indicate that these abnormalities are caused by activation of the sympathoadrenal system since they can largely be prevented by beta blockade [6]. We have proposed that these changes might cause ventricular tachycardia and account for the increased risk of sudden overnight death in young people with 
Type 1 diabetes [7], a condition which has been termed the 'dead-in-bed' syndrome [8]. Since hypoglycaemia induced QT lengthening can be prevented by beta-blocking agents then if we could establish which individuals are at particular risk it might prove possible to protect them using this therapeutic approach.

However before embarking on such a strategy it is important to establish whether QT intervals also lengthen during spontaneous clinical episodes of hypoglycaemia. It is possible that changes in the electrocardiogram observed using an experimental model of hypoglycaemia are artefactual since circulating insulin concentrations are higher and counterregulatory responses are more intense than during clinical episodes. It is as yet uncertain whether the QT interval is altered during hypoglycaemic episodes which occur with subcutaneous insulin therapy.

The aim of our study was to test the hypothesis that lengthening of the QTc interval occurs during spontaneous nocturnal hypoglycaemia in individuals with Type 1 diabetes.

\section{Subjects and methods}

Subjects. We recruited 22 patients with Type 1 diabetes. This sample size had a greater than $90 \%$ power to detect a mean increase of QT interval of $40 \mathrm{~ms}$ or more during hypoglycaemic nights assuming that hypoglycaemia occurred in $30 \%$ of the monitoring periods. Average age of the patients was $40.4 \pm 17.2$ years, mean duration of diabetes $17.2 \pm 9.3$ years, mean $\mathrm{HbA}_{1 \mathrm{c}}$ $8.2 \pm 1.2 \%$.

Of the subjects, 10 had no complications, one had maculopathy, three background retinopathy, two had hypertension, one subject a previous myocardial infarction, and one erectile dysfunction. All subjects gave written consent to participate in this study, which was approved by the North Sheffield Local Research ethics committee.

Subjects were admitted to the clinical investigation unit at 10 p.m. They took their usual bedtime insulin, along with any pre-bedtime snack, as would be part of their normal routine at home. A cannula was placed into an antecubital arm vein so that blood samples could be taken at hourly intervals and patients were encouraged to sleep. Blood was taken hourly, centrifuged in an adjacent room, and stored for later measurement of catecholamines and serum potassium. ECG electrodes were applied for recording of bipolar orthogonal (X, Y and Z) high resolution ECG's.

Measurement of $Q T$ interval. ECGs were recorded on a custom-built system for High-Resolution ECG Analysis that simultaneously acquires three bipolar orthogonal $\mathrm{X}, \mathrm{Y}$ and $\mathrm{Z}$ leads as previously described [4]. The ECG signal was recorded over a 10 min period onto a Racal Store 4 FM analogue tape recorder (Racal Instruments, Irvine, Calif., USA). These data were digitised using an Amplicon PC27 AD card (Amplicon Liveline, Brighton, UK) and a Toshiba desktop PC (Toshiba, Tokyo, Japan). The data were filtered and then averaged until the mean residual noise was below $1.5 \mu \mathrm{V}$; averaging of 10 to 30 beats at a rate of one every $6 \mathrm{~s}$ was required to achieve that figure. Only complexes with a correlation coefficient greater than $99.5 \%$ were accepted in the average. The period of each measurement took approximately 6-7 $\mathrm{min}$. The QT interval was measured in the averaged $\mathrm{X}, \mathrm{Y}$ and $\mathrm{Z}$ signals individually. QT interval was measured, using a tangent drawn at the point of maximal gradient on the downward slope of the $\mathrm{T}$ wave, and the end of the $\mathrm{T}$ wave marked as the point of intersection of the tangent with the isoelectric line [9]. The QT intervals were marked using an on-screen cursor by an observer who was blinded both to the prevailing blood glucose concentration and to the outcome of previous measurements.

The $\mathrm{X}, \mathrm{Y}$ and $\mathrm{Z}$ readings were then averaged to obtain an overall measure of cardiac repolarization.

Increases in heart rate are associated with a physiological reduction in the QT interval. We corrected for differences in heart rate, dividing QT by the square root of the RR interval, the Bazett correction, which mathematically normalises the QT interval to a heart rate of 60 beats/min [10].

Metabolic measurements. Plasma adrenaline was analysed by high performance liquid chromatography with electrochemical detection [11]. Serum potassium was analysed by direct potentiometry (Vitros Analyzer, Johnson and Johnson, Ortho Clinical Diagnostics, Haversham, Bucks., UK).

Statistics and analysis. Results are expressed as mean \pm SD. ECG recordings, and blood samples were taken at 11 p.m. and labelled as 'baseline'. If blood glucose level fell below $2.5 \mathrm{mmol} / \mathrm{l}$ at any point during the night, these were classified as 'nocturnal hypoglycaemia' nights. Nights where blood glucose concentration remained greater than $3.5 \mathrm{mmol} / \mathrm{l}$ were classed as 'nocturnal no hypoglycaemia'. Blood samples were taken and ECG's recorded hourly, with the QTc interval defined as the maximum of that recorded at glucose nadir, or glucose nadir $+1 \mathrm{~h}$. Of the seven subjects who became hypoglycaemic, the time of hypoglycaemia was 4 a.m. in three cases, 5 a.m. in two cases and midnight and $3 \mathrm{a} . \mathrm{m}$. in the other two. QT interval exhibits circadian rhythmicity with highest values between 1 a.m. and 5 a.m. [12]. We therefore selected $4 \mathrm{a} . \mathrm{m}$. as the start time for control data during non-hypoglycaemia nights in which we recorded the ECG for similar periods to those recorded during hypoglycaemic nights.

Differences between the overnight hypoglycaemic and nonhypoglycaemic periods were compared with unpaired $t$-tests.

\section{Results}

Of the 22 subjects with Type 1 diabetes, seven (32\%) developed hypoglycaemia. The remaining 15 subjects experienced a blood glucose concentration greater than $4 \mathrm{mmol} / \mathrm{l}$ during the night (Fig. 1). One subject had a blood glucose level of $2.9 \mathrm{mmol} / \mathrm{l}$ at 11 p.m., and subsequent readings all greater than $4 \mathrm{mmol} / \mathrm{l}$; for this subject baseline readings were recorded at 8 a.m. For all other subjects baseline ECG recordings and blood samples were taken at 11 p.m.

During hypoglycaemia QTc interval increased by $27 \mathrm{~ms}( \pm 15)$ from baseline (Fig. 2), compared with $9 \mathrm{~ms}( \pm 19)$ during no hypoglycaemia nights $(p=0.034$, $95 \% \mathrm{CI} 2,35)$. Adrenaline increased by $0.33 \mathrm{nmol}$ $( \pm 0.21)$ from baseline during overnight hypoglycaemia (Fig. 3), compared with $-0.05 \mathrm{nmol}( \pm 0.08)$ during no hypoglycaemia $(p=0.001,95 \%$ CI $0.19,0.56)$. There was no relationship between change in QTc and 


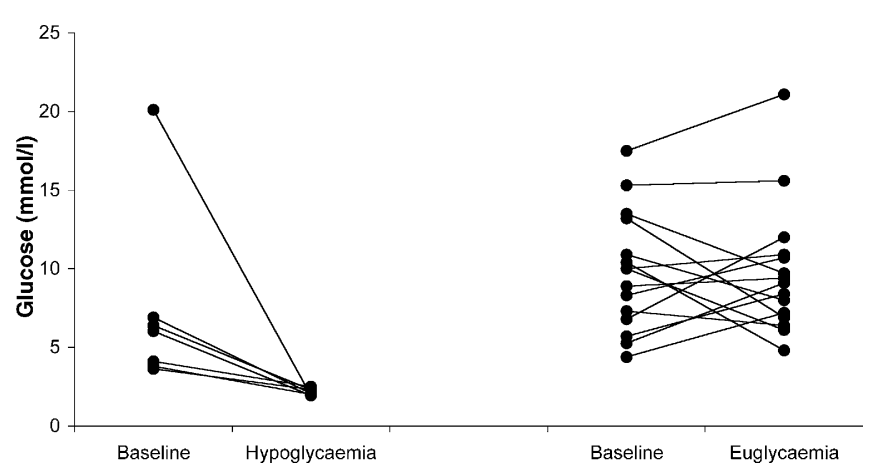

Fig. 1. Glucose concentration at baseline and during overnight hypoglycaemia and non-hypoglycaemia, $p=0.022$ between groups

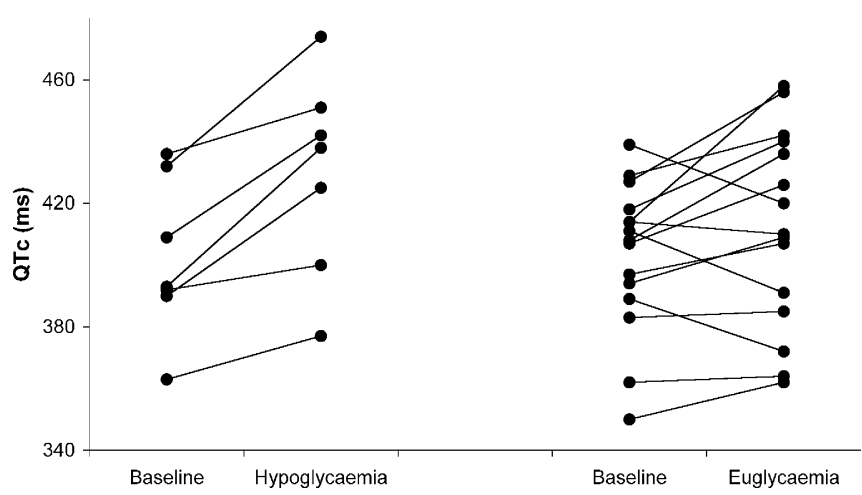

Fig. 2. QTc interval at baseline and during overnight hypoglycaemia and non-hypoglycaemia, $p=0.034$ between groups

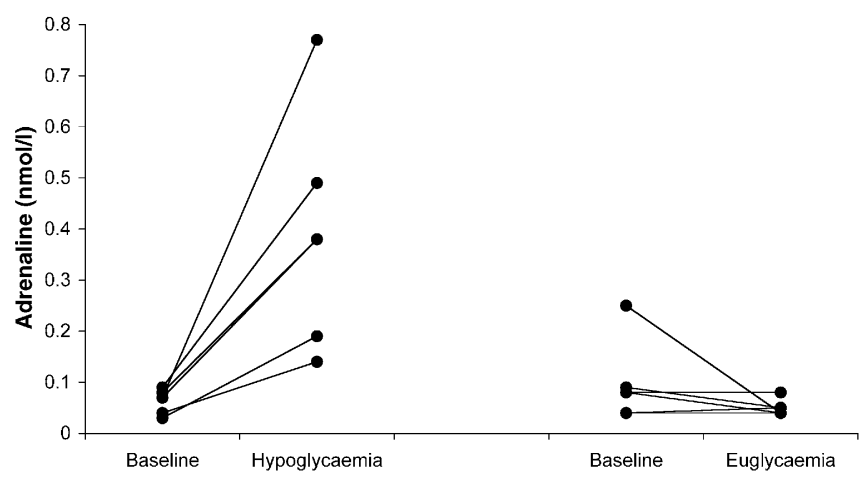

Fig. 3. Adrenaline concentration at baseline and during overnight hypoglycaemia and non-hypoglycaemia, $p=0.001$ between groups

magnitude of the adrenergic response during overnight hypoglycaemia $(\mathrm{r}=-0.012, p=0.982)$.

During hypoglycaemic nights, potassium fell $0.21 \mathrm{mmol} / \mathrm{l}( \pm 0.21)$ from baseline (Fig. 4), compared with $0.13 \mathrm{mmol} / \mathrm{l}( \pm 0.39)$ during no hypoglycaemia $(p=0.59,95 \% \mathrm{CI}-0.25,0.42)$. There was no relationship between change in QTc and change in potassium $(\mathrm{r}=0.725, p=0.065)$. Noradrenaline fell by $0.28 \mathrm{nmol} / 1( \pm 0.94)$ from baseline during overnight hypoglycaemia, compared with $0.41 \mathrm{nmol} / \mathrm{l}$

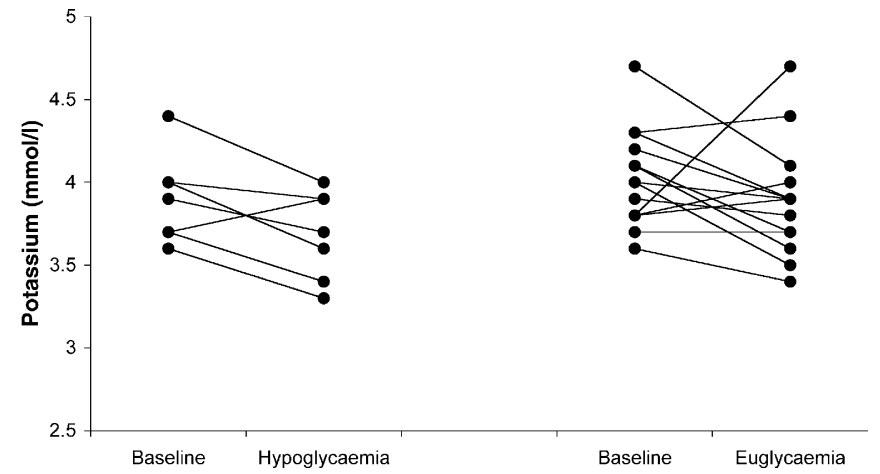

Fig. 4. Potassium concentration at baseline and during overnight hypoglycaemia and non-hypoglycaemia, $p=0.59$ between groups

$( \pm 0.51)$ during no hypoglycaemia $(p=0.76,95 \%$ CI $-0.77,1.03)$.

\section{Discussion}

These data show that significant albeit modest increases in QTc interval are associated with 'clinical' periods of hypoglycaemia developing spontaneously during the night. The mean increases in QTc of $27 \mathrm{~ms}$ over baseline and maximum increases of $45 \mathrm{~ms}$ are smaller than we observed during experimental hypoglycaemia where the mean increase was around $75 \mathrm{~ms}$. Furthermore, cardiac repolarization has a circadian rhythm and normally lengthens during the night. It is therefore important to consider whether the changes we observed merely reflect physiological alterations in QT interval and are without clinical relevance. We were careful to control for circadian lengthening of QT interval by measuring QTc during nonhypoglycaemic nights at similar times to hypoglycaemic nights and observed longer QTc intervals during hypoglycaemia. Furthermore the modest increases in QTc reflect the attenuated sympathoadrenal response during the relatively mild hypoglycaemic episodes we observed during the study.

We have recently shown in non-diabetic subjects, that the increases in QTc interval during hypoglycaemia seem to be due to sympathoadrenal activation from both a direct effect and also indirectly due to catecholamine-induced lowering of serum potassium [6]. Catecholamine responses during hypoglycaemia were between five to ten times greater in those experimental studies compared to the increases we observed during the spontaneous nocturnal episodes in the present work. Furthermore, experimental hypoglycaemia in subjects with either Type 1 [4] or Type 2 diabetes [13] provokes a more intense sympathoadrenal response and larger increases in QT interval. It is therefore not surprising that in the present study, the degree of QT lengthening was also lower. Nevertheless, it is consistent with our hypothesis that abnormal cardiac repo- 
lorization and hypoglycaemia-induced sympathoadrenal activation are related although the correlation between the increase in QTc and adrenaline was not significant. Interestingly in this study we found an apparently stronger association (albeit statistically insignificant) between change in QTc and change in potassium concentration suggesting that this mechanism (either directly or indirectly) could also play a role.

A modest counterregulatory response during nocturnal hypoglycaemia was not unexpected. The data confirm previous work which showed diminished counterregulatory responses during clinical nocturnal episodes in both children [14] and adults [15], particularly during REM sleep [16]. Our study population had a relatively long duration of diabetes which is associated with impaired glucose counterregulation [17].

The clinical relevance of our findings remains uncertain, particularly since these subjects did not develop severe hypoglycaemia and a strong sympathoadrenal response. Nevertheless, we believe our data are consistent with the hypothesis that abnormal cardiac repolarization can develop during spontaneous hypoglycaemia. On occasions individuals with insulintreated diabetes experience powerful counterregulatory responses during clinical episodes, reporting intense autonomic symptoms when waking during severe nocturnal hypoglycaemia. It is possible that during such attacks, when sympathoadrenal activation is at its most powerful that a potentially hazardous degree of QT lengthening could develop, perhaps in individuals with unrecognised congenital QT lengthening or when taking particular medications known to prolong the QT interval [18] including alcohol [19].

It seems to us worthwhile undertaking more extensive ECG monitoring during clinical episodes of nocturnal hypoglycaemia in patients with Type 1 diabetes, perhaps using recently available methods of continuous glucose monitoring. Such an approach might identify more severe hypoglycaemic episodes and establish whether this results in prolonged and potentially hazardous QT lengthening.

Acknowledgements. We thank M. Cunningham, S. Hudson and R. Macneil for expert technical assistance. This work was supported by a Project Grant from Diabetes UK (formerly the British Diabetic Association).

\section{References}

1. Dessertenne F (1966) Ventricular tachycardia with 2 variable opposing foci. Arch Mal Coeur Vaiss 59:263-272

2. Curran ME, Splawski I, Timothy KW et al. (1995) A molecular basis for cardiac arrhythmias: HERG mutations cause long QT syndrome. Cell 80:795-803
3. Schouten EG, Dekker JM, Meppelink P et al. (1991) QT interval prolongation predicts cardiovascular mortality in an apparently healthy population. Circulation 84:15161523

4. Marques JL, George E, Peacey SR et al. (1997) Altered ventricular repolarization during hypoglycaemia in patients with diabetes. Diabet Med 14:648-654

5. Robinson RT, Harris ND, Ireland RH, Lindholm A, Heller SR (2003) Comparative effect of human soluble insulin and insulin aspart upon hypoglycaemia-induced alterations in cardiac repolarization. Br J Clin Pharmacol 55:246251

6. Robinson RT, Harris ND, Ireland RH et al. (2003) Mechanisms of abnormal cardiac repolarization during insulininduced hypoglycemia. Diabetes 52:1469-1474

7. Harris ND, Heller SR (1999) Sudden death in young patients with Type 1 diabetes: a consequence of disease, treatment or both? Diabet Med 16:623-625

8. Campbell IW (1991) Dead in bed syndrome:a new manifestation of nocturnal hypoglycaemia? Diabet Med 8: 3-4

9. Ireland RH, Robinson RT, Heller SR, Marques JL, Harris ND (2000) Measurement of high resolution ECG QT interval during controlled euglycaemia and hypoglycaemia. Physiol Meas 21:295-303

10. Bazett HC (1920) An analysis of the time relations of electrocardiograms. Heart 7:353-370

11. Forster CD, Macdonald IA (1999) The assay of the catecholamine content of small volumes of human plasma. Biomed Chromatogr 13:209-215

12. Ong JJ, Sarma JS, Venkataraman K, Levin SR, Singh BN (1993) Circadian rhythmicity of heart rate and QTc interval in diabetic autonomic neuropathy: implications for the mechanism of sudden death. Am Heart J 125:744-752

13. Landstedt-Hallin L, Englund A, Adamson U, Lins PE (1999) Increased QT dispersion during hypoglycaemia in patients with type 2 diabetes mellitus. J Intern Med 246: 299-307

14. Matyka KA, Crowne EC, Havel PJ et al. (1999) Counterregulation during spontaneous nocturnal hypoglycemia in prepubertal children with type 1 diabetes. Diabetes Care 22:1144-1150

15. Bendtson I, Rosenfalck AM, Binder C (1993) Nocturnal versus diurnal hormonal counterregulation to hypoglycemia in type 1 (insulin-dependent) diabetic patients. Acta Endocrinol 128:109-115

16. Jones TW, Porter P, Sherwin RS et al. (1998) Decreased epinephrine responses to hypoglycemia during sleep. $\mathrm{N}$ Engl J Med 338:1657-1662

17. Bolli GB, De Feo P, Compagnucci P et al. (1983) Abnormal glucose counterregulation in IDDM. Interaction of anti-insulin antibodies and impaired glucagon and epinephrine secretion. Diabetes 32:134-141

18. De Ponti F, Poluzzi E, Vaccheri A et al. (2002) Non-antiarrhythmic drugs prolonging the QT interval: considerable use in seven countries. Br J Clin Pharmacol 54:171177

19. Rossinen J, Sinisalo J, Partanen J, Nieminen MS, Viitasalo M (1999) Effects of acute alcohol infusion on duration and dispersion of QT interval in male patients with coronary artery disease and in healthy controls. Clin Cardiol 22: 591-594 